\title{
Formation of AICl by radiative association
}

\author{
C. M. Andreazza, ${ }^{1 \star}$ A. A. de Almeida ${ }^{2 \star}$ and R. M. Vichietti ${ }^{3 \star}$ \\ ${ }^{1}$ Universidade Estadual Paulista, IGCE, DEMAC, Av. 24A, 1515, CEP 13506-700, Rio Claro, SP, Brazil \\ ${ }^{2}$ Universidade de São Paulo, IAG, Rua do Matão, 1226, CEP 05508-090, São Paulo, SP, Brazil \\ ${ }^{3}$ Universidade de São Paulo, IQSC, Av. Trabalhador São-carlense, 400, CEP 13566-590, São Carlos, SP, Brazil
}

Accepted 2018 March 2. Received 2018 January 26; in original form 2016 December 27

\begin{abstract}
The rate coefficient for the formation of aluminium monochloride, $\mathrm{AlCl}$, from the radiative association of aluminium and chlorine atoms is estimated as a function of temperature. The coupling of the $\mathrm{Al}$ and $\mathrm{Cl}$ atoms through the $\mathrm{A}^{1} \Pi$ molecular electronic state, which undergoes radiative transition to the $\mathrm{X}^{1} \Sigma^{+}$ground state, is the most efficient transition to form $\mathrm{AlCl}$. The rate constant was found to vary with temperature according to the expressions $k(T)=1.22 \times 10^{-16}(T / 300)^{0.40} \exp (-748 / T) \mathrm{cm}^{3} \mathrm{~s}^{-1}$ for temperatures between 300 and $1000 \mathrm{~K}$, and $k(T)=2.20 \times 10^{-16}(T / 300)^{0.175} \exp (-1067 / T) \mathrm{cm}^{3} \mathrm{~s}^{-1}$ for temperatures between 1000 and $14000 \mathrm{~K}$.
\end{abstract}

Key words: atomic data-atomic processes-circumstellar matter-ISM: molecules.

\section{INTRODUCTION}

Aluminium monochloride $(\mathrm{AlCl})$ has been spectroscopically identified in the circumstellar material of the carbon-rich $(\mathrm{C} / \mathrm{O}$ abundance ratio > 1) star IRC+10216 (Cernicharo \& Guélin 1987; Highberger $\&$ Ziurys 2003). The central low-mass asymptotic giant branch (AGB) star IRC+10216 has an effective temperature of around 2000-3500 K (e.g. Truong-Bach, Morris \& Nguyen-Q-Rieu 1991; Groenewegen, van der Veen \& Matthews 1998). It has been suggested that this star pulsates due to instabilities in its interior and material flows from the stellar surface (Iben \& Renzini 1983) at a rate of 1-4 × $10^{-5} \mathrm{M}_{\odot} \mathrm{yr}^{-1}$ (Truong-Bach, Morris \& Nguyen-QRieu 1991; Groenewegen et al. 1998). The mass loss and radiation pressure on dust produce an extended envelope (up to several astronomical units). In addition, searches for $\mathrm{AlCl}$ in the carbon-rich post-AGB stars CRL 2688 and CRL 618 have been unsuccessful (Highberger \& Ziurys 2003). Thus, the $\mathrm{AlCl}$ vanishes during the post-AGB phase of evolution, but the mechanisms are not known (e.g. Highberger \& Ziurys 2003).

AGB stars have reached the last stage of stellar evolution. The dredge-up of newly formed carbon produced by triple- $\alpha$ burning in the stellar interior may change the less massive progenitors (1.0 4.0 $\mathrm{M}_{\odot}$ ) into C-rich stars (Herwig 2005). In contrast, for the most massive progenitors $\left(4.0-8.0 \mathrm{M}_{\odot}\right)$, the hot-bottom burning favours the conversion of carbon to nitrogen via the $\mathrm{CN}$-cycle, resulting in less carbon than oxygen in their circumstellar shells (Vassiliadis \& Wood 1993; Herwig 2005).

$\mathrm{AlCl}$ has also been found in the oxygen-rich material around the red supergiant star VY CMa (Kamiński et al. 2013). Its effective

^E-mail: carmenma@rc.unesp.br (CMA); amaury.almeida@rc.unesp.br (AAdeA); vichietti_rm@yahoo.com.br(RMV) temperature is near 3200-4000 K (Massey, Levesque \& Plez 2006; Wittkowski et al. 2012). Shocks due to pulsation of the star can drive the mass-loss rate of $0.5 \times 10^{-4}$ to $3.0 \times 10^{-3} \mathrm{M}_{\odot} \mathrm{yr}^{-1}$ (Monnier et al. 1999; Wittkowski et al. 2012). This outflow leads to the formation of an extended circumstellar shell ( $\sim 7-14 \mathrm{au})$ around the star.

Moreover, AlNC and AlF have also been found in the C-rich star IRC+10216 (Cernicharo \& Guélin 1987; Ziurys, Apponi \& Phillips 1994; Ziurys et al. 2002), and $\mathrm{AlO}$ and $\mathrm{AlOH}$ have been discovered in O-rich red supergiant VY CMa, as well as in O-rich AGB star $o$ Cet (Tenenbaum \& Ziurys 2009, 2010; Kamiński et al. 2016). Furthermore, AlH and neutral atomic aluminium have also been detected towards $o$ Cet (Kamiński et al. 2016). According to theoretical models, the condensation temperature of Al-bearing compounds starts around $1700 \mathrm{~K}$ (Lodders 2003). At around $1000 \mathrm{~K}$, aluminium is entirely accreted on to dust grains (Lodders \& Fegley 1995). Thus, the Al-containing molecules appear to be restricted to the warm inner layers of the C- and O-rich shell of evolved stars (Ziurys et al. 1994; Kamiński et al. 2013). However, AlNC and a relatively high abundance of $\mathrm{AlCl}$ and $\mathrm{AlF}$ are observed far away from the star IRC+10216 (Agúndez et al. 2012). It has been suggested that in the outer layers of the envelope, these molecules are survivors of the previous inner region chemistry or that grains are destroyed by shock waves and release Al to later recreate the Al-containing molecules (Highberger \& Ziurys 2003).

Chemical equilibrium calculations predict an abundance of $\mathrm{AlCl}$ close to those observed (Tenenbaum \& Ziurys 2009; Agúndez et al. 2012). On the other hand, these models do not appear to reproduce the observed $\mathrm{AlO}$ abundance in the O-rich star VY CMa (Tenenbaum \& Ziurys 2009). The non-equilibrium chemistry in the inner shell of the $\mathrm{C}$-rich evolved stars appears to form $\mathrm{AlCl}$ very efficiently (Cherchneff 2012), but AIF is not considered in this study. Moreover, the non-equilibrium chemical processes explain the presence of the $\mathrm{AlO}$ in the inner region of O-rich circumstellar shells, 
although it fails to explain the formation of $\mathrm{AlCl}$ (Gobrecht et al. 2016). In the inner layers of the envelope, chemical compounds can be formed by radiative association (Cherchneff 2012). The rate constant for the formation of $\mathrm{AlCl}$ by radiative association in $\mathrm{Al}$ and $\mathrm{Cl}$ collisions is not known. Here, we present estimates of the rate constant for the radiative association reaction $\mathrm{Al}+\mathrm{Cl} \rightarrow \mathrm{AlCl}+\mathrm{h} v$ in the temperature range between 300 and $14000 \mathrm{~K}$, which could be of some interest in the innermost region of the circumstellar shells of evolved stars or other astronomical environments, such as supernovae and novae ejecta.

\section{RADIATIVE ASSOCIATION}

Radiative association is a chemical process, $\mathrm{A}+\mathrm{B} \rightarrow \mathrm{AB}+\mathrm{h} v$, in which an intermediate unstable complex $\mathrm{AB} *$ is formed by collision of the A and B species. During the interaction, this complex can be stabilized against redissociation by emission of a photon, forming a stable $\mathrm{AB}$ molecule. The rate constant of association $k$ is defined by $\mathrm{d} n(\mathrm{AB}) / \mathrm{d} t=k n(\mathrm{~A}) n(\mathrm{~B})$, where $n(\mathrm{AB}), n(\mathrm{~A})$ and $n(\mathrm{~B})$ are the number density of $\mathrm{AB}, \mathrm{A}$ and $\mathrm{B}$ species (e.g. Bates 1951).

The rate coefficient $k(E)$ for a two-body collision with incident energy $E$ is

$k(E)=v(E) \sigma(E)$,

where $v(E)$ is the velocity of the incident particles and $\sigma(E)$ is the radiative cross-section, which is obtained following the semiclassical scheme used in previous works (e.g. Andreazza \& de Almeida 2014; Andreazza, de Almeida \& Borin 2016). This semiclassical formalism was developed by Bates (1951) and may be applied to radiative coupling of massive reactants (Bates 1951; Zygelman \& Dalgarno 1988), which form tightly bound products (Gustafsson 2013). In this description, the cross-section can be written as

$\sigma(E)=4 \pi g\left(\frac{\mu}{2 E}\right)^{1 / 2} \int_{0}^{\infty} b \mathrm{~d} b \int_{r_{c}}^{\infty} \frac{A(r) \mathrm{d} r}{\left[1-\frac{b^{2}}{r^{2}}-\frac{V^{\prime}(r)}{E}\right]^{1 / 2}}$,

where $\mu$ is the reduced mass of the colliding particles, $r_{c}$ is the classical turning point, $r$ is the internuclear distance, $b$ is the impact parameter and $V^{\prime}(r)$ is the initial or upper interatomic potential. $g$ is the ratio of the statistical weight for the initial or upper molecular electronic state to the sum of the statistical weights of all molecular electronic states correlating with the same separated atomic states, and is given by

$g=\frac{\left(2 S^{\prime}+1\right)\left(2-\delta_{0, \Lambda^{\prime}}\right)}{\left(2 L_{\mathrm{A}}+1\right)\left(2 S_{\mathrm{A}}+1\right)\left(2 L_{\mathrm{B}}+1\right)\left(2 S_{\mathrm{B}}+1\right)}$,

where $L_{\mathrm{A}}, S_{\mathrm{A}}, L_{\mathrm{B}}$ and $S_{\mathrm{B}}$ are the electronic orbital and electronic spin angular momenta of the A and B atoms, respectively. $S^{\prime}$ is the total electronic spin of the upper molecular state, $\Lambda^{\prime}$ is the projection of the electronic orbital angular momentum of the upper molecular state on to the internuclear axis and $A(r)$ is the radial spontaneous emission coefficient given by

$A(r)=\frac{64 \pi^{4} g^{\prime}}{3 h^{4} c^{3}}\left|V^{\prime}(r)-V^{\prime \prime}(r)\right|^{3}|D(r)|^{2} \mathrm{~s}^{-1}$,

in which $c$ is the speed of light, $V^{\prime \prime}(r)$ is the lower interatomic potential, $D(r)$ is the radial transition moment and $g^{\prime}$ is the statistical weight factor given by

$g^{\prime}=\frac{\left(2-\delta_{0, \Lambda^{\prime}+\Lambda^{\prime \prime}}\right)}{\left(2-\delta_{0, \Lambda^{\prime}}\right)}$,

where $\Lambda^{\prime \prime}$ is the projection of the electronic orbital angular momentum of the lower molecular state on to the internuclear axis. The factor $g^{\prime}$ is equal to 2 for $\Sigma \rightarrow \Pi$ transitions and equal to unity for all other transitions.

The thermal rate constant $k(T)$ is obtained at temperature $T$ assuming a Maxwell-Boltzmann distribution for the relative velocities of the colliding reactants:

$k(T)=\left(\frac{8}{\mu \pi}\right)^{1 / 2}\left(\frac{1}{k_{\mathrm{B}} T}\right)^{3 / 2} \int_{0}^{\infty} E \sigma(E) \exp \left(-\frac{E}{k_{\mathrm{B}} T}\right) \mathrm{d} E$,

where $k_{\mathrm{B}}$ is the Boltzmann constant.

\section{RESULTS AND DISCUSSION}

In the inner layers of the circumstellar material of evolved stars, which have a temperature of between 2000 and $4000 \mathrm{~K}$, only molecular electronic states correlating to the lowest energy asymptote need to be considered. Thus, the coupling of $\mathrm{Al}\left({ }^{2} \mathrm{P}\right)$ and $\mathrm{Cl}\left({ }^{2} \mathrm{P}\right)$ atoms gives rise to singlet and triplet molecular states of symmetries $\Delta, \Pi$ (two states), $\Sigma^{+}$(two states) and $\Sigma^{-}$.

The first experimental observation of the $\mathrm{A}^{1} \Pi-\mathrm{X}^{1} \Sigma^{+}$transition of $\mathrm{AlCl}$ was presented by Bhaduri \& Fowler (1934). This system was later confirmed by Holst (1935) and analysed at high resolution by Mahieu, Dubois \& Bredohl (1989a). Sharma (1951) reported the identification of the $\mathrm{a}^{3} \Pi-\mathrm{X}^{1} \Sigma^{+}$and $\mathrm{b}^{3} \Sigma^{+}-\mathrm{a}^{3} \Pi$ systems. Highresolution studies of the $\mathrm{a}^{3} \Pi-\mathrm{X}^{1} \Sigma^{+}$transition of $\mathrm{AlCl}$ have also been done by Ram et al. (1982), Mahieu, Dubois \& Bredohl (1989b) and Saksena, Dixit \& Singh (1998). The $b^{3} \Sigma^{+}-a^{3} \Pi$ system has also been reported by Mahieu et al. (1989b) and they reassigned the $\mathrm{b}^{3} \Sigma^{+}$state as $\mathrm{b}^{3} \Delta$.

The interatomic potentials of the $\mathrm{A}^{1} \Pi$ and $\mathrm{X}^{1} \Sigma^{+}$molecular electronic states and the electronic transition moment connecting these two states were theoretically found by Langhoff, Bauschlicher \& Taylor (1988) using the multireference configuration interaction (MRCI) method. They also computed accurate spectroscopic constants for the lowest six singlet $\left(\mathrm{X}^{1} \Sigma^{+}, \mathrm{A}^{1} \Pi, 1^{1} \Sigma^{-}, 2^{1} \Sigma^{+}, 1^{1} \Delta\right.$ and $\left.2^{1} \Pi\right)$ and the lowest five triplet $\left(\mathrm{a}^{3} \Pi, \mathrm{b}^{3} \Sigma^{+}, 2^{3} \Sigma^{+}, 2^{3} \Pi\right.$ and $1^{3} \Delta$ ) molecular electronic states as well as the vibrational lifetimes of these states. Their calculations are in excellent agreement with experiments. The molecular constants and rovibrational lifetimes of the $\mathrm{A}^{1} \Pi$ state, and the interatomic potentials for the lowest states of $\mathrm{AlCl}$ have also been calculated by Brites, Hammoutène \& Hochlaf (2008) at the MRCI level of theory. Both works confirm the early attribution of the $\mathrm{b}^{3} \Sigma^{+}$state rather than to $\mathrm{b}^{3} \Delta$.

The coupling of the $\mathrm{Al}\left({ }^{2} \mathrm{P}\right)$ and $\mathrm{Cl}\left({ }^{2} \mathrm{P}\right)$ atoms can proceed through the excited $1^{1} \Sigma^{-}, 2^{1} \Sigma^{+}, 1^{1} \Delta, 2^{1} \Pi, \mathrm{b}^{3} \Sigma^{+}, 2^{3} \Sigma^{+}, 2^{3} \Pi, 1^{3} \Delta$ and $1^{3} \Sigma^{-}$molecular electronic states of AlCl. The $1^{1} \Sigma^{-}, 2^{1} \Sigma^{+}, 1^{1} \Delta$, $2^{1} \Pi, 2^{3} \Pi, 1^{3} \Delta$ and $1^{3} \Sigma^{-}$states are repulsive (Brites et al. 2008). Hence, these last entrance channels make little contribution to the rate constant. In addition, the potential curves of the $b^{3} \Sigma^{+}$and $2^{3} \Sigma^{+}$ molecular electronic states exhibit minima above the first atomic dissociation limit, to which they dissociate. Thus, the contribution of the $2^{3} \Sigma^{+}-b^{3} \Sigma^{+}, 2^{3} \Sigma^{+}-a^{3} \Pi$ and $b^{3} \Sigma^{+}-a^{3} \Pi$ transitions to the radiative association is expected to be very small.

The radiative recombination of $\mathrm{Al}\left({ }^{2} \mathrm{P}\right)$ and $\mathrm{Cl}\left({ }^{2} \mathrm{P}\right)$ atoms can also proceed through the excited $\mathrm{A}^{1} \Pi$ molecular electronic state, which undergoes a radiative transition to the ground $\mathrm{X}^{1} \Sigma^{+}$state in a time-scale of the order of $6.4 \pm 2.5 \mathrm{~ns}$ from $v^{\prime}=0$ (Rogowski $\&$ Fontijn 1987). For this, we use the accurate interatomic potentials of the $\mathrm{A}^{1} \Pi$ and $\mathrm{X}^{1} \Sigma^{+}$molecular states and the corresponding electronic transition moment reported by Langhoff et al. (1988). In the short internuclear region, the interatomic potential curves and 


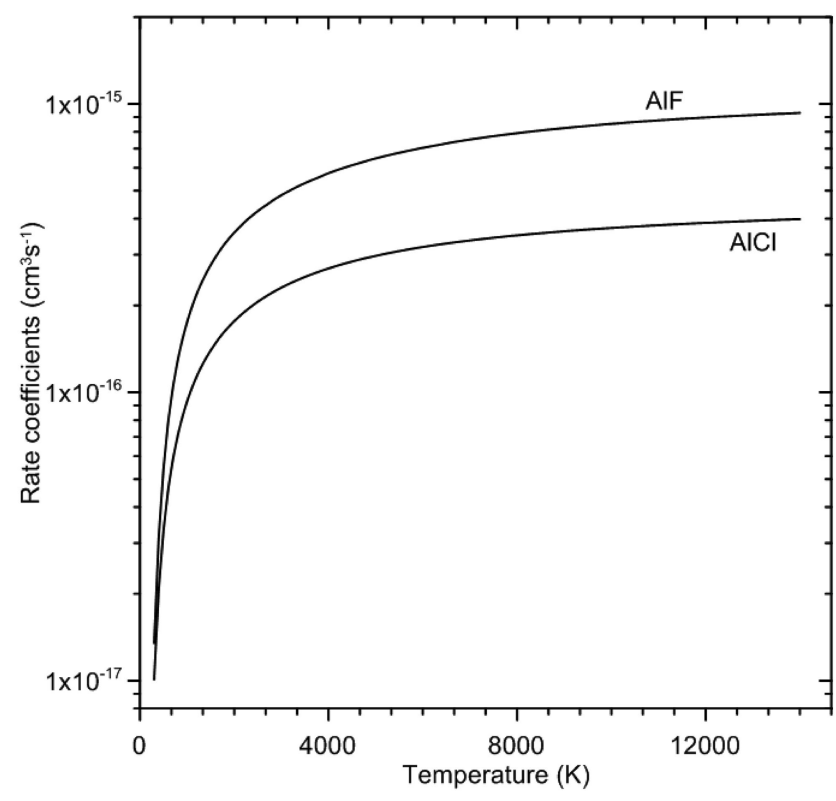

Figure 1. Rate constants for the radiative association of $\mathrm{Al}\left({ }^{2} \mathrm{P}\right)$ and $\mathrm{Cl}\left({ }^{2} \mathrm{P}\right)$ and of $\mathrm{Al}\left({ }^{2} \mathrm{P}\right)$ and $\mathrm{F}\left({ }^{2} \mathrm{P}\right)$ to form $\mathrm{AlCl}$ and $\mathrm{AlF}$, respectively, both via the $\mathrm{A}^{1} \Pi$ state.

transition moment function were fitted (Dalgarno, Kirby \& Stancil 1996; Stancil et al. 1997) to the forms $V(r)=a \exp (-b r)+c$ and $R_{e}(r)=d r^{2}+e r$, respectively. For the $\mathrm{A}^{1} \Pi-\mathrm{X}^{1} \Sigma^{+}$transition, the statistical weight factor $g=2 / 3$.

The computed reaction rate for the formation of $\mathrm{AlCl}$ through the $\mathrm{A}^{1} \Pi-\mathrm{X}^{1} \Sigma^{+}$system is shown in Fig. 1, which is approximated to within 3 per cent by the expressions

$k(T)=1.22 \times 10^{-16}\left(\frac{T}{300}\right)^{0.4} \exp \left(\frac{-748}{T}\right) \mathrm{cm}^{3} \mathrm{~s}^{-1}$

for temperatures between 300 and $1000 \mathrm{~K}$, and

$k(T)=2.20 \times 10^{-16}\left(\frac{T}{300}\right)^{0.175} \exp \left(\frac{-1067}{T}\right) \mathrm{cm}^{3} \mathrm{~s}^{-1}$

for temperatures between 1000 and $14000 \mathrm{~K}$.

Because of the existence of a repulsive hump in the potential energy curve of the $\mathrm{A}^{1} \Pi$ molecular electronic state of $\mathrm{AlCl}$, the rate constant decreases quickly at very low temperatures. However, the barrier height in the $\mathrm{AlCl} \mathrm{A}^{1} \Pi$ interatomic potential calculated by Langhoff et al. (1988) appears to be smaller than that in fig. 2 of Brites et al. (2008). At low temperatures, both semiclassical and quantum calculations depend on the potential barrier height. Hence, uncertainties in the height of the barrier introduce a significant error in the rate constant at low temperatures. In addition, we do not take the atomic fine-structure levels of the aluminium and chlorine atoms into account. The maximum value of the atomic fine-structure splitting of $\mathrm{Al}$ and $\mathrm{Cl}$ atoms is around $994 \mathrm{~cm}^{-1}$ or $1430 \mathrm{~K}$, consequently uncertainties are expected in our results below $1500 \mathrm{~K}$. Moreover, semiclassical calculations excluded quantum tunnelling and shape resonance (Bain \& Bardsley 1972; Smith 1989; Gustafsson 2013). It is difficult to estimate the uncertainties in our calculations. The computed reaction rate has an uncertainty that decreases with increasing temperature. Thus, we suggest that there is an uncertainty of one order of magnitude at the lowest temperatures. In the hightemperature rate constant, an uncertainty of about a factor of two to three is expected.
The rate constant for the formation of AlF, through the coupling of the $\mathrm{Al}\left({ }^{2} \mathrm{P}\right)$ and $\mathrm{F}\left({ }^{2} \mathrm{P}\right)$ atoms (Andreazza \& de Almeida 2014), is also shown for comparison in Fig. 1. AlF is also formed via the $\mathrm{A}^{1} \Pi-\mathrm{X}^{1} \Sigma^{+}$transition. The $\mathrm{A}^{1} \Pi$ molecular electronic state of the AlF molecule also haso a repulsive hump. The barrier is larger for AlF (Langhoff et al. 1988). Hence, the rate constant decreases more rapidly at low temperature for AlF. For the temperatures found in the innermost regions of an evolved star (2000 to $4000 \mathrm{~K})$, the radiative rate constant for the formation of AlF is higher than that for the $\mathrm{Al}+\mathrm{Cl}$ reaction. This difference is due to higher transition dipole moments and the greater transition frequencies involved in the $\mathrm{A}-\mathrm{X}$ system of AlF.

\section{CONCLUSIONS}

The rate constant for the formation of $\mathrm{AlCl}$ via the radiative association of $\mathrm{Al}\left({ }^{2} \mathrm{P}\right)$ and $\mathrm{Cl}\left({ }^{2} \mathrm{P}\right)$ atoms has been estimated. Inspection of the calculated potential energy curves (Langhoff et al. 1988; Brites et al. 2008) indicates that radiative association occurs mainly via the $\mathrm{A}^{1} \Pi$ molecular electronic state accompanied by radiative transition to the $\mathrm{X}^{1} \Sigma^{+}$ground state.

In inner regions of evolved stars, which have temperatures between 2000 and $4000 \mathrm{~K}$, the radiative recombination of $\mathrm{Al}$ and $\mathrm{Cl}$ atoms to produce $\mathrm{AlCl}$ is relatively slow. This result indicates that only a modest amount of $\mathrm{AlCl}$ can be synthesized by radiative association close to the photospheres of AGBs and red supergiant stars.

\section{ACKNOWLEDGEMENTS}

We are grateful to Fundação de Amparo à Pesquisa do Estado de São Paulo (FAPESP), Brazil, and the Conselho Nacional de Desenvolvimento Científico e Tecnológico (CNPq), Brazil, for academic support.

\section{REFERENCES}

Agúndez M., Fonfría J. P., Cernicharo J., Kahane C., Daniel F., Guélin M., 2012, AA, 543, A48

Andreazza C. M., de Almeida A. A., 2014, MNRAS, 437, 2932

Andreazza C. M., de Almeida A. A., Borin A. C., 2016, MNRAS, 457, 3096

Bain R. A., Bardsley J. N., 1972, J. Phys. B Atom. Molec. Phys., 5, 277

Bates D. R., 1951, MNRAS, 111, 303

Bhaduri B. N., Fowler A., 1934, Proc. R. Soc. Lond. Ser. A, 145, 321

Brites V., Hammoutène D., Hochlaf M., 2008, J. Phys. Chem. A, 112, 13419

Cernicharo J., Guélin M., 1987, AA, 183, L10

Cherchneff I., 2012, AA, 545, 12

Dalgarno A., Kirby K., Stancil P. C., 1996, ApJ, 458, 397

Gobrecht D., Cherchneff I., Sarangi A., Plane J. M. C., Bromley S. T., 2016, AA, 585, A6

Groenewegen M. A. T., van der Veen W. E. C. J., Matthews H. E., 1998, AA, 338, 491

Gustafsson M., 2013, J. Chem. Phys., 138, 074308

Herwig F., 2005, ARA\&A, 43, 435

Highberger J. L., Ziurys L. M., 2003, ApJ, 597, 1065

Holst W., 1935, Z. f. Phys., 93, 55

Iben I., Jr, Renzini A., 1983, ARAA, 21, 271

Kamiński T., Gottlieb C. A., Young K. H., Menten K. M., Patel N. A., 2013, ApJSS, 209, 38

Kamiński T. et al., 2016, AA, 592, 42K

Langhoff S. R., Bauschlicher C. W., Jr, Taylor P. R., 1988, J. Chem. Phys., 88,5715

Lodders K., 2003, ApJ, 591, 1220

Lodders K., Fegley B., Jr, 1995, Meteoritics, 30, 661 
Mahieu E., Dubois I., Bredohl H., 1989a, J. Mol. Spectrosc., 134, 317

Mahieu E., Dubois I., Bredohl H., 1989b, J. Mol. Spectrosc., 138, 264

Massey P., Levesque E. M., Plez B., 2006, ApJ, 646, 1203

Monnier J. D., Tuthill P. G., Lopez B., Cruzalebes P., Danchi W. C., Haniff C. A., 1999, ApJ, 512, 351

Ram R. S., Rai S. B., Upadhya K. N., Rai D. K., 1982, Phys. Scr., 26, 383

Rogowski D. F., Fontijn A., 1987, Chem. Phys. Lett., 137, 219

Saksena M. D., Dixit V. S., Singh M., 1998, J. Mol. Spectrosc., 187, 1

Sharma D., 1951, ApJ, 113, 210

Smith I. W. M., 1989, ApJ, 347, 282

Stancil P. C., Kirby K., Sannigrahi A. B., Buenker R. J., Hirsch G., Gu J.-P., 1997, ApJ, 486, 574

Tenenbaum E. D., Ziurys L. M., 2009, ApJ, 694, L59
Tenenbaum E. D., Ziurys L. M., 2010, ApJ, 712, L93

Truong-Bach, Morris D., Nguyen-Q-Rieu, 1991, AA, 249, 435

Vassiliadis E., Wood P. R., 1993, ApJ, 413, 641

Wittkowski M., Hauschildt P. H., Arroyo-Torres B., Marcaide J. M., 2012, AA, 540, L12

Ziurys L. M., Apponi A. J., Phillips T. J., 1994, ApJ, 433, 729

Ziurys L. M., Savage C., Highberger J. L., Apponi A. J., 2002, ApJ, 564, L45

Zygelman B., Dalgarno A., 1988, Phys. Rev. A, 38, 1877

This paper has been typeset from a $\mathrm{T}_{\mathrm{E}} \mathrm{X} / \mathrm{LAT} \mathrm{T}$ file prepared by the author. 